\title{
Electroweak relaxation of cosmological hierarchy
}

\author{
Shao-Jiang Wang* \\ Tufts Institute of Cosmology, Department of Physics and Astronomy, Tufts University, \\ 574 Boston Avenue, Medford, Massachusetts 02155, USA
}

(Received 19 October 2018; published 28 January 2019)

\begin{abstract}
A simple model for the late-time cosmic acceleration problem is presented in the Starobinsky inflation with a negative bare cosmological constant as well as a nonminimal coupling to the Higgs boson. After electroweak symmetry breaking, the Starobinsky inflaton has been frozen until very recently, becoming a thawing quintessence, and a comparable magnitude to the observed dark energy density can be achieved without fine-tuning. Our proposal essentially reduces the cosmological constant problem into the electroweak hierarchy problem, and its late-time behavior is also consistent with the recently proposed swampland criteria.
\end{abstract}

DOI: $10.1103 /$ PhysRevD.99.023529

\section{INTRODUCTION}

Although physics at different energy scales are decoupled from each other according to the renormalization group (RG) flow, the energy scales themselves could still reveal some connections among physics at these scales. It has long been noticed that [1] the energy scale of the currently observed dark energy density $\Lambda_{\mathrm{DE}}^{4} \sim\left(10^{-12} \mathrm{GeV}\right)^{4}$ could be expressed as

$$
\Lambda_{\mathrm{DE}}^{2} \sim H_{0} M_{\mathrm{Pl}},
$$

where the Planck scale $M_{\mathrm{Pl}} \sim 10^{18} \mathrm{GeV}$ is the smallest ultraviolet (UV) length scale and the current Hubble scale $H_{0} \sim 10^{-42} \mathrm{GeV}$ is the largest infrared (IR) length scale. This suspicious UV/IR mixing relation has inspired some quests $[2,3]$ for the late-time cosmic acceleration problem [4,5]. The same pattern is also realized for inflation with a form

$$
\Lambda_{\mathrm{inf}}^{2} \sim M_{\mathrm{Pl}} H_{\mathrm{inf}}
$$

that can be recognized trivially as the Friedmann equation. The face values $\Lambda_{\text {inf }} \sim 10^{16} \mathrm{GeV}$ and $H_{\text {inf }} \sim 10^{14} \mathrm{GeV}$ could be inferred from the current constraint [6] on the tensor-toscalar ratio $r \lesssim 0.01$.

A similar relation was observed recently in [7] that

$$
v_{\mathrm{EW}}^{2} \sim \Lambda_{\mathrm{DE}} \Lambda_{\mathrm{inf}},
$$

*schwang@cosmos.phy.tufts.edu

Published by the American Physical Society under the terms of the Creative Commons Attribution 4.0 International license. Further distribution of this work must maintain attribution to the author(s) and the published article's title, journal citation, and DOI. Funded by SCOAP ${ }^{3}$. where the electroweak (EW) hierarchy $v_{\mathrm{EW}} \sim 10^{2} \mathrm{GeV}$ and cosmological hierarchy $\Lambda_{\mathrm{DE}}^{4} \sim\left(10^{-12} \mathrm{GeV}\right)^{4}$ are conspired via an inflationary scale $\Lambda_{\text {inf }} \sim 10^{16} \mathrm{GeV}$ without fine-tuning. Therefore, the question of why the observed cosmological constant is extremely small could be transformed into the question of why the measured EW scale is relatively small. The cosmological hierarchy problem can thus be solved as long as a solution to the EW hierarchy problem is known prior.

In the spirit of quintessential inflation [8], a concrete example [7] to reproduce the relation (3) is constructed in a nonstandard Starobinsky inflation model with a noncanonical kinetic term and a nonstandard Higgs potential,

$$
\begin{aligned}
S= & \int \mathrm{d}^{4} x \sqrt{-g}\left\{\frac{M_{\mathrm{Pl}}^{2}}{2} R-\frac{1}{2}\left[1+\left(\frac{h}{v}\right)^{2}\left(b \frac{M_{\mathrm{Pl}}}{\phi}\right)^{2}\right](\partial \phi)^{2}\right. \\
& \left.-\alpha^{2} M_{\mathrm{Pl}}^{4}\left(1-\mathrm{e}^{-\sqrt{\frac{2}{3} M_{\mathrm{Pl}}}}\right)^{2}-V(h)\left[1+c\left(\mathrm{e}^{-\sqrt{\frac{8}{3} \phi}}-1\right)\right]\right\},
\end{aligned}
$$

where $\alpha=9.97 \times 10^{-6}$ is fixed from the observation. After Starobinsky inflation and subsequent reheating, the inflaton remnant stays at a minimal slightly shifted from the origin due to a nonzero Higgs potential value $V(0)=\frac{\lambda}{4} v_{\mathrm{EW}}^{4}$ in a symmetric phase with $\lambda=0.129$. Once the EW symmetry is broken, the Higgs is relaxed to the EW vacuum and the inflaton is frozen by a dubbed bait-and-switch mechanism at a potential energy density

$$
c^{2} \frac{V(h=0)^{2}}{\alpha^{2} M_{\mathrm{Pl}}^{4}}=\frac{c^{2} \lambda^{2} v^{8}}{16 \alpha^{2} M_{\mathrm{Pl}}^{4}}=4 c^{2} \times 10^{-48} \mathrm{GeV}^{4}
$$

that could match the currently observed dark energy density $\rho_{\Lambda} \sim 2.58 \times 10^{-47} \mathrm{GeV}^{4}$ for $c \approx 2.5$ without fine-tuning. 
After that, the frozen inflaton starts rolling down a quintessential potential when the Hubble parameter drops down to its current value. Unfortunately, the model in [7] has the serious drawback that, at the quantum level, the noncanonical kinetic term $(h / v)^{2}(\partial \phi / \phi)^{2}$ is only suppressed by the EW scale, leaving observable signals that would otherwise have been detected in the Higgs decay channels a long time ago. Furthermore, the construction in (5) seems highly nontrivial and unnatural.

In this paper, a simple and natural model to reproduce the relation (3) is constructed in the standard Starobinsky inflation model [9] with a negative bare cosmological constant as well as a nonminimal coupling to the Higgs boson. The general picture of [7] is retained without the use of any noncanonical kinetic term and nonstandard Higgs potential. The comparable magnitude to the observed dark energy density can be achieved without fine-tuning thanks to the relation (3). Our proposal is also consistent with the recently proposed swampland criteria [10-12] due to the transformed role of the inflaton as a thawing quintessence at late time.

\section{THE MODEL}

The action of our proposal in the Jordan frame is

$$
\begin{aligned}
S_{J}= & \int \mathrm{d}^{4} x \sqrt{-g}\left[\frac{M^{2}}{2}\left(1+\frac{R}{8 \alpha^{2} M^{2}}+\frac{\xi h^{2}}{M^{2}}\right) R-\Lambda_{b}^{4}\right. \\
& \left.-\frac{1}{2}(\partial h)^{2}-V(h)-\mathcal{L}_{\mathrm{SM}+\mathrm{DM}}\right],
\end{aligned}
$$

where $M$ is an unknown energy scale for $R^{2}$ gravity to be fixed later; $\alpha$ is an inflationary parameter to be fixed by observation; $\xi$ is a nonminimal coupling of the Higgs field $h$ to the Ricci scalar $R$ that eventually will be generated at loop order even if it is absent at tree level [13], $\Lambda_{b}$ is a bare cosmological constant that turns out to be negative later; and $V(h)$ is the usual Higgs potential of the form

$$
V(h)= \begin{cases}\frac{\lambda}{4} h^{4}+\frac{\lambda}{4} v^{4}, & \text { symmetric phase } \\ \frac{\lambda}{4}\left(h^{2}-v^{2}\right)^{2}, & \text { broken phase }\end{cases}
$$

with $\lambda=0.13$. The Lagrangian $\mathcal{L}_{\mathrm{SM}+\mathrm{DM}}$ for the SM along with an unknown dark matter (DM) sector will be left implicitly thereafter. See, e.g., [14-16] for similar actions but in different contexts, and in particular [17] for a comprehensive study on $\alpha$-attractor quintessential inflation.

The Starobinsky scalaron $s$ is introduced as an auxiliary field to rewrite (6) as

$$
\begin{aligned}
S_{J}= & \int \mathrm{d}^{4} x \sqrt{-g}\left[\frac{M^{2}}{2}\left(1+\frac{s}{4 \alpha^{2} M^{2}}+\frac{\xi h^{2}}{M^{2}}\right) R-\frac{s^{2}}{16 \alpha^{2}}\right. \\
& \left.-\Lambda_{b}^{4}-\frac{1}{2}(\partial h)^{2}-V(h)\right],
\end{aligned}
$$

so that its equation-of-motion (EOM) $s=R$ could recover the original form (6). Einstein gravity is recovered at $\left(s=s_{0}, h=v\right)$ if

$$
M^{2}=M_{\mathrm{Pl}}^{2}-\frac{s_{0}}{4 \alpha^{2}}-\xi v^{2},
$$

and the action in the Jordan frame becomes

$$
\begin{aligned}
S_{J}= & \int \mathrm{d}^{4} x \sqrt{-g}\left[\frac{M_{\mathrm{Pl}}^{2}}{2} \Omega(s, h)^{2} R-\frac{1}{2}(\partial h)^{2}\right. \\
& \left.-\frac{s^{2}}{16 \alpha^{2}}-\Lambda_{b}^{4}-V(h)\right],
\end{aligned}
$$

where a conformal factor

$$
\Omega(s, h)^{2}=1+\frac{s-s_{0}}{4 \alpha^{2} M_{\mathrm{Pl}}^{2}}+\xi \frac{h^{2}-v^{2}}{M_{\mathrm{Pl}}^{2}}
$$

is introduced to transform the metric to be $\tilde{g}_{\mu \nu}=\Omega^{2} g_{\mu \nu}$ so that the action in the Einstein frame is obtained as of the form

$$
\begin{aligned}
S_{E}= & \int \mathrm{d}^{4} x \sqrt{-\tilde{g}}\left[\frac{M_{\mathrm{Pl}}^{2}}{2} \tilde{R}-\frac{1}{2}\left(M_{\mathrm{Pl}} \sqrt{\frac{3}{2}} \ln \Omega^{2}\right)^{2}\right. \\
& \left.-\frac{1}{2} \frac{(\tilde{\partial} h)^{2}}{\Omega^{2}}-\frac{s^{2}}{16 \alpha^{2} \Omega^{4}}-\frac{\Lambda_{b}^{4}+V(h)}{\Omega^{4}}\right] .
\end{aligned}
$$

By introducing the scalar fields $\psi_{h}=M_{\mathrm{Pl}} \sqrt{\frac{3}{2}} \ln \Omega(s, h)^{2}$, the action in the Einstein frame could be expressed as of the form

$$
\begin{aligned}
S_{E}= & \int \mathrm{d}^{4} x \sqrt{-\tilde{g}}\left\{\frac{M_{\mathrm{Pl}}^{2}}{2} \tilde{R}-\frac{1}{2}\left(\tilde{\partial} \psi_{h}\right)^{2}-\frac{1}{2} \mathrm{e}^{-\sqrt{\frac{2}{3} \psi_{\mathrm{Pl}}}}(\tilde{\partial} h)^{2}\right. \\
& -\alpha^{2} M_{\mathrm{Pl}}^{4}\left[1-\mathrm{e}^{-\sqrt{\frac{2}{3} \psi_{\mathrm{Pl}}}}\left(1+\xi \frac{h^{2}-v^{2}}{M_{\mathrm{Pl}}^{2}}-\frac{s_{0}}{4 \alpha^{2} M_{\mathrm{Pl}}^{2}}\right)\right]^{2} \\
& \left.-\mathrm{e}^{-\sqrt{\frac{\sqrt{3}}{3} M_{\mathrm{Pl}}}}\left[\Lambda_{b}^{4}+V(h)\right]\right\} .
\end{aligned}
$$

As you will see, $\phi \equiv \psi_{0}$ is the inflaton before EW symmetry breaking and $\varphi \equiv \psi_{v}$ is the quintessence after EW symmetry breaking. For the sake of simplicity, we will get rid of the tilde symbol and use the following short notations,

$$
\omega_{h}^{2}=1+\xi \frac{h^{2}-v^{2}}{M_{\mathrm{Pl}}^{2}}, \quad \omega_{0}^{2}=1-\frac{\xi v^{2}}{M_{\mathrm{Pl}}^{2}}, \quad \omega_{v}^{2}=1 ;
$$

$S=\frac{s}{4 \alpha^{2} M_{\mathrm{Pl}}^{2}}, \quad S_{0}=\frac{s_{0}}{4 \alpha^{2} M_{\mathrm{Pl}}^{2}}, \quad \Omega_{h}^{2}(S)=S-S_{0}+\omega_{h}^{2} ;$

$U_{h}=\frac{\Lambda_{b}^{4}+V(h)}{\alpha^{2} M_{\mathrm{Pl}}^{4}}, \quad U_{0}=\frac{\Lambda_{b}^{4}}{\alpha^{2} M_{\mathrm{Pl}}^{4}}+\frac{V(0)}{\alpha^{2} M_{\mathrm{Pl}}^{4}} \equiv U_{v}+V_{0}$, 
to express the action in the Einstein frame as

$$
\begin{aligned}
S_{E}= & \int \mathrm{d}^{4} x \sqrt{-g}\left[\frac{M_{\mathrm{Pl}}^{2}}{2} R-\frac{1}{2}\left(\partial \psi_{h}\right)^{2}-\frac{1}{2} \Omega_{h}^{-2}(\partial h)^{2}\right. \\
& \left.-\alpha^{2} M_{\mathrm{Pl}}^{4} \frac{U_{h}+S^{2}}{\left(S-S_{0}+\omega_{h}^{2}\right)^{2}}\right],
\end{aligned}
$$

where the potential term in the second line will be denoted as $W(S, h)$.

\section{STAROBINSKY INFLATION}

To have a successful Starobinsky inflation before EW symmetry breaking, $\omega_{0}^{2}-S_{0}$ in (13) should be positive, and thus $\omega_{0}^{2}-S_{0}=\exp \left(\sqrt{\frac{2}{3}} c\right)$ for some constant $c$. Furthermore, the bare cosmological constant term in (17) should not interfere with the end of inflation roughly at $\phi_{\text {end }} / M_{\mathrm{Pl}}=1+c$, namely,

$$
\left(\Lambda_{b}^{4}+V(0)\right) \mathrm{e}^{-2 \sqrt{\frac{2}{3}}(1+c)} \ll \alpha^{2} M_{\mathrm{Pl}}^{4}\left(1-\mathrm{e}^{-\sqrt{\frac{2}{3}}}\right)^{2},
$$

leading to a constraint

$$
\frac{U_{0}}{\left(\omega_{0}^{2}-S_{0}\right)^{2}} \ll\left(\mathrm{e}^{\sqrt{\frac{2}{3}}}-1\right)^{2} \approx 1.6
$$

that will be checked later. Another constraint comes from the suppression of fluctuations in the Higgs sector to reserve the inflationary prediction of Starobinsky inflation. This requires the effective mass of the kinetically normalized Higgs $\chi$ from $(\mathrm{d} h / \mathrm{d} \chi)^{2}=\Omega_{h}^{2}$ to be larger than the inflationary Hubble scale,

$$
\begin{aligned}
m_{\chi}^{2} & =\Omega_{0}^{2} W_{h}^{\prime \prime}(S, 0)=\frac{-4 \xi}{M_{\mathrm{Pl}}^{2}}\left(\alpha^{2} M_{\mathrm{Pl}}^{4} \frac{S^{2}}{\Omega_{0}^{4}(S)}+\frac{\Lambda_{b}^{4}+V(0)}{\Omega_{0}^{4}(S)}\right) \\
& \approx \frac{-4 \xi S^{2}}{\Omega_{0}^{4}(S)} \alpha^{2} M_{\mathrm{Pl}}^{2} \gg H_{\mathrm{inf}}^{2}=\frac{S^{2}}{3 \Omega_{0}^{4}(S)} \alpha^{2} M_{\mathrm{Pl}}^{2}
\end{aligned}
$$

namely, $|\xi| \gg 1 / 12$, which will also be checked later. For now, we will assume that these two constraints are satisfied so that Starobinsky inflation can proceed as usual.

After Starobinsky inflation and subsequent reheating, the inflaton remnant, if it does not decay away totally, stays at a local minimum $\left(S_{\mathrm{EW}}, h=0\right)$ determined from the condition

$$
\frac{W_{\phi}^{\prime}\left(S_{\mathrm{EW}}, 0\right)}{\alpha^{2} M_{\mathrm{Pl}}^{4}}=\sqrt{\frac{8}{3}} \frac{1}{M_{\mathrm{Pl}}} \frac{S_{\mathrm{EW}}\left(\omega_{0}^{2}-S_{0}\right)-U_{0}}{\Omega_{0}^{4}\left(S_{\mathrm{EW}}\right)}=0,
$$

which gives rise to the field value of the Starobinsky scalaron just before EW symmetry breaking,

$$
S_{\mathrm{EW}}=\frac{U_{0}}{\omega_{0}^{2}-S_{0}}
$$

\section{EW SYMMETRY BREAKING}

When EW symmetry is broken, the Higgs is relaxed to its current minimum $h=v$ and the potential energy density is of the form

$$
W\left(S_{\mathrm{EW}}, v\right)=\alpha^{2} M_{\mathrm{Pl}}^{4} \frac{U_{v}+S_{\mathrm{EW}}^{2}}{\left(1-S_{0}+S_{\mathrm{EW}}\right)^{2}} .
$$

To retain the success of the picture observed in [7], $\varphi$ should be frozen right after EW symmetry breaking by requiring a light effective mass of $\varphi$,

$$
\begin{aligned}
m_{\varphi}^{2} & =W_{\varphi}^{\prime \prime}\left(S_{\mathrm{EW}}, v\right) \\
& =\frac{4}{3} \alpha^{2} M_{\mathrm{Pl}}^{2} \frac{2 U_{v}+\left(1-S_{0}\right)\left(1-S_{0}-S_{\mathrm{EW}}\right)}{\left(1-S_{0}+S_{\mathrm{EW}}\right)^{2}}
\end{aligned}
$$

If the observation from relation (3) indeed reveals the myth of dark energy, all we have to do is to solve the fixing condition (23) and freezing condition (24), namely,

$$
\begin{gathered}
a V_{0}^{2}=\frac{U_{v}+S_{\mathrm{EW}}^{2}}{\left(1-S_{0}+S_{\mathrm{EW}}\right)^{2}} \\
b V_{0}^{2}=\frac{2 U_{v}+\left(1-S_{0}\right)\left(1-S_{0}-S_{\mathrm{EW}}\right)}{\left(1-S_{0}+S_{\mathrm{EW}}\right)^{2}} .
\end{gathered}
$$

To match the currently observed dark energy density $W\left(S_{\mathrm{EW}}, v\right) \sim \Lambda_{\mathrm{DE}}^{4}$ and thawing behavior $m_{\varphi}^{2} \sim H_{0}^{2}$, one only needs for the order-of-one parameters $a=25 / 4$ and $b=a / 4 \Omega_{\Lambda}$, with $\Omega_{\Lambda} \approx 0.7$ today.

Solving (25) and (26) is a nontrivial task. The only freedom comes from the normalized scalaron value $S_{0}$, where Einstein gravity is fixed. By choosing $S_{0}$ away from 1 , one expects the following approximated solutions:

$$
\begin{gathered}
\omega_{0}^{2} \approx \frac{3}{2} S_{0}-\frac{1}{2} ; \\
U_{v} \approx-\frac{1}{4}\left(S_{0}-1\right)^{2} .
\end{gathered}
$$

However, these solutions do not allow for the desirable behavior at both early time and late time that necessarily requires $\omega_{0}^{2}-S_{0}>0$ and $1-S_{0}>0$ from (13). 


\section{THAWING QUINTESSENCE}

It turns out as a nice surprise that, when $S_{0}$ is close to $1^{-}$, the position of $S_{0}$ with desirable solutions is independent of the parameters $a$ and $b$. To see this, one could take a concrete example by choosing $S_{0}=1-V_{0}$ without lost of generality. Equations (25) and (26) are solved to give

$$
\begin{gathered}
\omega_{0}^{2}=3-\frac{3}{2} V_{0}+(3 b-6 a) V_{0}^{2}+\mathcal{O}\left(V_{0}^{3}\right) \\
U_{v}=-\frac{1}{4} V_{0}^{2}+\frac{3}{4}(a+b) V_{0}^{4}+\mathcal{O}\left(V_{0}^{6}\right),
\end{gathered}
$$

that are truncated at the order when parameters $a$ and $b$ first appear. The leading order terms of (29) and (30) are indeed independent of the choice of how close $S_{0}$ is to $1^{-}$. The potentials in (13) along the $\psi_{h}$ direction in the symmetric and broken phase are presented in Fig. 1 with the above truncated solutions from $S_{0}=1-V_{0}$, where the EW symmetry breaking occurs in the normalized scalaron value $S_{\mathrm{EW}}=V_{0} / 2+(6 a-3 b) V_{0}^{3} / 4+\mathcal{O}\left(V_{0}^{5}\right)$, with $\Omega_{0}^{2}\left(S_{\mathrm{EW}}\right)=2+(3 b-6 a) V_{0}^{2}+\mathcal{O}\left(V_{0}^{3}\right)$ and $\Omega_{v}^{2}\left(S_{\mathrm{EW}}\right)=$ $3 V_{0} / 2+(6 a-3 b) V_{0}^{3} / 4+\mathcal{O}\left(V_{0}^{5}\right), \quad$ namely, $\quad \phi_{\mathrm{EW}}=$ $\sqrt{3 / 2} \ln \Omega_{0}^{2}\left(S_{\mathrm{EW}}\right)=0.8489 M_{\mathrm{Pl}}$ and $\varphi_{\mathrm{EW}}=\sqrt{3 / 2}$ $\ln \Omega_{v}^{2}\left(S_{\mathrm{EW}}\right)=-151.788 M_{\mathrm{Pl}}$. The broken-phase potential is thus shifted appropriately for clarity. The normalized scalaron value at final $\mathrm{AdS}$ minimum in the broken phase is $S_{\min }=-V_{0} / 4+3(a+b) V_{0}^{3} / 4+\mathcal{O}\left(V_{0}^{5}\right)$ with $\Omega_{v}^{2}\left(S_{\min }\right)=3 V_{0} / 4+3(a+b) V_{0}^{3} / 4+\mathcal{O}\left(V_{0}^{5}\right), \quad$ namely, $\varphi_{\min }=\sqrt{3 / 2} \ln \Omega_{v}^{2}\left(S_{\min }\right)=-152.637 M_{\mathrm{Pl}}$. Note that the rolling of $\varphi$ in the future $\Delta \varphi=\varphi_{\mathrm{EW}}-\varphi_{\min }=\phi_{\mathrm{EW}}$ is a sub-Planckian field excursion.

Using the truncated solutions (29) and (30), one can check that the original equations (25) and (26) are trivially satisfied at the leading order,

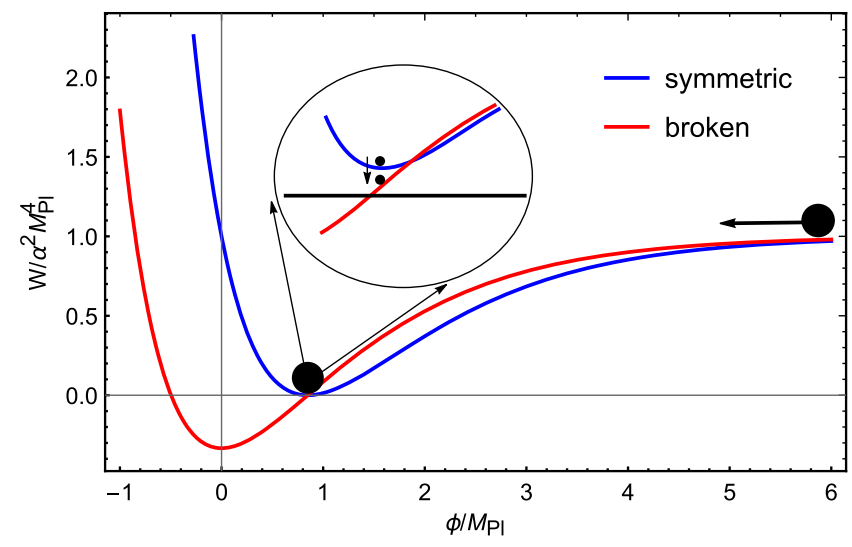

FIG. 1. The demonstration of the physical picture of our model from the truncated solutions (29) and (30) with $S_{0}=1-V_{0}$. The potential in (13) along the $\varphi$ direction in the broken phase has been shifted appropriately for illustration.

$$
\begin{aligned}
& \frac{U_{v}+S_{\mathrm{EW}}^{2}}{\left(1-S_{0}+S_{\mathrm{EW}}\right)^{2}}=a V_{0}^{2}+\frac{4 a+b}{12} V_{0}^{3}+\mathcal{O}\left(V_{0}^{4}\right) ; \\
& \frac{\frac{2 U_{v}}{\left(1-S_{0}\right)^{2}}+1-\frac{S_{\mathrm{EW}}}{1-S_{0}}}{\left(1+\frac{S_{\mathrm{EW}}}{1-S_{0}}\right)^{2}}=b V_{0}^{2}-\frac{4 a+b}{12} V_{0}^{3}+\mathcal{O}\left(V_{0}^{4}\right) \text {, }
\end{aligned}
$$

which freezes the inflaton at the right position after EW symmetry breaking with a potential energy density and effective mass

$$
\begin{gathered}
W\left(S_{\mathrm{EW}}, v\right)=a \frac{V(0)^{2}}{\alpha^{2} M_{\mathrm{Pl}}^{4}} \approx \Lambda_{\mathrm{DE}}^{4} ; \\
m_{\varphi}^{2}\left(S_{\mathrm{EW}}, v\right)=\frac{4 b}{3 M_{\mathrm{Pl}}^{2}} \frac{V(0)^{2}}{\alpha^{2} M_{\mathrm{Pl}}^{4}} \approx H_{0}^{2},
\end{gathered}
$$

desirable for our purpose. The Starobinsky inflaton is thus frozen until the Hubble parameter drops down to its current value and becoming a thawing quintessence today, which also explains the coincidence problem.

One can also check the early-time behavior from the truncated solutions (29) and (30). During inflation, the constraint (19) is explicitly satisfied:

$$
\frac{U_{0}}{\left(\omega_{0}^{2}-S_{0}\right)^{2}}=\frac{1}{4} V_{0}+\frac{1}{16} V_{0}^{2}+\mathcal{O}\left(V_{0}^{3}\right) \ll 1.6 .
$$

The other constraint (20), or equivalently $|\xi| \gg 1 / 12$, is also explicitly satisfied from (29), namely,

$$
-\xi=\frac{M_{\mathrm{Pl}}^{2}}{v^{2}}\left(2-\frac{3}{2} V_{0}+\mathcal{O}\left(V_{0}^{2}\right)\right) \approx 10^{32} \gg \frac{1}{12} .
$$

Note that the constraint [18] on $|\xi| \lesssim 10^{15}$ is not applicable here due to the presence of $R^{2}$ gravity in addition to the nonminimal coupling. The decay channel of the Higgs to quintessence from the coupling term $\exp \left(-\sqrt{\frac{2}{3}} \varphi / M_{\mathrm{Pl}}\right)(\partial h)^{2}$ is highly suppressed by the Planck scale, leaving no trace in the collider. The Planckian suppressed effect on various couplings in the SM potential also evades the bounds from the fifth force. The large effective mass of the Higgs during inflation could protect it from the dangerous quantum kick into the unwanted large-field minimum. The Higgs instability problem (see, e.g., $[19,20]$ for a brief review) is thus cured as a by-product.

\section{SWAMPLAND CRITERIA}

The standard single-field slow-roll inflationary paradigm currently faces some tension [21] with the original de Sitter conjecture in the swampland criteria $[10,11]$ as well as the refined de Sitter conjecture [12] that either one of the following conditions, 


$$
\begin{aligned}
|\nabla V| & \geq \frac{c}{M_{\mathrm{Pl}}} V ; \\
\min \left(\nabla_{i} \nabla_{j} V\right) & \leq-\frac{c^{\prime}}{M_{\mathrm{Pl}}^{2}} V,
\end{aligned}
$$

is fulfilled for some universal constants $c, c^{\prime}>0$ of order 1 . Here $V$ is a potential of scalar fields $\phi_{i}$ in a low energy effective theory of any consistent quantum gravity, and the minimum eigenvalue in the second condition is taken for the Hessian operator $\nabla_{i} \nabla_{j} V$ in an orthonormal frame. See also [22-29] for possible ways out of swampland, [30-48] for the implications, and [49-58] for the debates.

Although our action (6) contains a bare cosmological constant, which turns out to be mildly negative deduced from (30),

$$
\Lambda_{b}^{4} \approx-\frac{1}{4} \frac{V(0)^{2}}{\alpha^{2} M_{\mathrm{Pl}}^{4}} \sim-\Lambda_{\mathrm{DE}}^{4},
$$

the plateau potential is currently in tension with the swampland criteria, unless turning to, for example, warm inflation [27,29] or non-Bunch-Davies initial states [24,28]. Nevertheless, the late-time behavior of our proposal is consistent with the original de Sitter conjecture in the swampland criteria due to the transformed role of the Starobinsky inflaton as a thawing quintessence with

$$
\begin{aligned}
M_{\mathrm{Pl}} \frac{\left|\nabla_{\varphi} W\left(S_{\mathrm{EW}}, v\right)\right|}{W\left(S_{\mathrm{EW}}, v\right)} & =\sqrt{\frac{8}{3}} \frac{\left(1-S_{0}\right) S_{\mathrm{EW}}-U_{v}}{S_{\mathrm{EW}}^{2}+U_{v}} \\
& \approx \frac{2}{3 a} \sqrt{\frac{2}{3}} V_{0}^{-2} \gg \mathcal{O}(1),
\end{aligned}
$$

while

$$
\begin{aligned}
M_{\mathrm{Pl}}^{2} \frac{\min \left(\nabla_{i} \nabla_{j} W\right)}{W\left(S_{\mathrm{EW}}, v\right)} & =M_{\mathrm{Pl}}^{2} \frac{\nabla_{\varphi} \nabla_{\varphi} W}{W\left(S_{\mathrm{EW}}, v\right)} \\
& =\frac{4}{3} \frac{\left(1-S_{0}\right)\left(1-S_{0}-S_{\mathrm{EW}}\right)+2 U_{v}}{S_{\mathrm{EW}}^{2}+U_{v}} \\
& \approx \frac{4 b}{3 a}=\frac{1}{3 \Omega_{\Lambda}} .
\end{aligned}
$$

The future destiny of our Universe is starting to roll down the quintessential potential, eventually crossing the zero point of the potential and inevitably approaching the final $\mathrm{AdS}$ minimum with potential energy density

$W\left(S_{\min }, v\right)=\alpha^{2} M_{\mathrm{Pl}}^{4} \frac{U_{v}+\left(\frac{U_{v}}{1-S_{0}}\right)^{2}}{\left(1-S_{0}+\frac{U_{v}}{1-S_{0}}\right)^{2}} \approx-\frac{1}{3} \alpha^{2} M_{\mathrm{Pl}}^{4}$

within one Planckian field excursion, $\Delta \varphi=\varphi_{\mathrm{EW}}-\varphi_{\min }=$ $\phi_{\mathrm{EW}} \approx 0.85 \times M_{\mathrm{Pl}}$, which is also consistent with the distance conjecture of the swampland criteria $[10,11]$.

\section{CONCLUSION}

To naturally reproduce the conspired relation among the interplay of the EW scale and the inflationary scale with the dark energy scale, we propose a simple model of quintessential Starobinsky inflation to address the late-time cosmic acceleration problem. The model in the Jordan frame is simply defined in $R^{2}$ gravity with a bare negative cosmological constant term as well as a nonminimal coupling of the Higgs to the Ricci scalar. When transformed into the Einstein frame, the Starobinsky inflation is obtained, and the Higgs instability problem is solved due to a large effective mass. After EW symmetry breaking, the Starobinsky inflaton is frozen at a potential energy density (33) comparable to the currently observed dark energy density without fine-tuning. Only until recently when the Hubble parameter drops down to its current value does the inflaton start rolling down a quintessential potential, eventually ending up in an AdS state within one Planckian field excursion. The late-time behavior is consistent with the recently proposed swampland criteria.

\section{DISCUSSION}

There are infinite truncated solutions to Eqs. (25) and (26) with similar leading order terms on the right-hand-side when $S_{0}$ is close to $1^{-}$, which might be regarded as a reflection of the string landscape at the effective-fieldtheory (EFT) level. Any solution with $S_{0}$ chosen to be away from 1 is in the regime of string swampland, where our observable Universe cannot be obtained. Even in the regime of the string landscape, a larger value of the EW scale than the currently measured value would either freeze the inflaton at such a large energy density that life cannot have enough time to form or be incapable of freezing the inflaton at all so that our Universe quickly rolls down to the final AdS minimum. Therefore, the anthropic principle for the EW hierarchy problem is thus implied. In this respect, although the cosmological constant problem can be naturally solved in light of relation (3) within our model, an explanation for a relatively small EW scale is still needed, for example, supersymmetry [59], extra dimensions $[60,61]$, strong dynamics $[62,63]$, cosmological relaxion [64], or $N$ naturalness [65].

\section{ACKNOWLEDGMENTS}

We are grateful to Alexander Vilenkin, Zhong-Zhi Xianyu, and Masaki Yamada for their useful and stimulating discussions. We also want to thank Tommi Markkanen for helpful correspondence. This work is supported by the postdoctoral scholarship of Tufts University. 
[1] A. G. Cohen, D. B. Kaplan, and A. E. Nelson, Effective Field Theory, Black Holes, and the Cosmological Constant, Phys. Rev. Lett. 82, 4971 (1999).

[2] S. D. H. Hsu, Entropy bounds and dark energy, Phys. Lett. B 594, 13 (2004).

[3] M. Li, A model of holographic dark energy, Phys. Lett. B 603, 1 (2004).

[4] A. G. Riess et al. (Supernova Search Team), Observational evidence from supernovae for an accelerating universe and a cosmological constant, Astron. J. 116, 1009 (1998).

[5] S. Perlmutter et al. (Supernova Cosmology Project), Measurements of Omega and Lambda from 42 high redshift supernovae, Astrophys. J. 517, 565 (1999).

[6] Y. Akrami et al. (Planck Collaboration), Planck 2018 results. X. Constraints on inflation, arXiv:1807.06211.

[7] K. Dimopoulos and T. Markkanen, Dark energy as a remnant of inflation and electroweak symmetry breaking, arXiv: 1807.04359.

[8] P. J. E. Peebles and A. Vilenkin, Quintessential inflation, Phys. Rev. D 59, 063505 (1999).

[9] A. A. Starobinsky, Spectrum of relict gravitational radiation and the early state of the universe, Pisma Zh. Eksp. Teor. Fiz. 30, 719 (1979) [JETP Lett. 30, 682 (1979)].

[10] T. D. Brennan, F. Carta, and C. Vafa, The string landscape, the Swampland, and the missing corner, Proc. Sci., TASI2017 (2017) 015 [arXiv:1711.00864].

[11] G. Obied, H. Ooguri, L. Spodyneiko, and C. Vafa, De Sitter space and the Swampland, arXiv:1806.08362.

[12] H. Ooguri, E. Palti, G. Shiu, and C. Vafa, Distance and de Sitter conjectures on the Swampland, Phys. Lett. B 788, 180 (2019).

[13] D. Z. Freedman, I. J. Muzinich, and E. J. Weinberg, On the energy-momentum tensor in gauge field theories, Ann. Phys. (N.Y.) 87, 95 (1974).

[14] Y.-C. Wang and T. Wang, Primordial perturbations generated by Higgs field and R2 operator, Phys. Rev. D 96, 123506 (2017).

[15] Y. Ema, Higgs scalaron mixed inflation, Phys. Lett. B 770, 403 (2017).

[16] M. He, A. A. Starobinsky, and J. Yokoyama, Inflation in the mixed Higgs-R2 model, J. Cosmol. Astropart. Phys. 05 (2018) 064.

[17] Y. Akrami, R. Kallosh, A. Linde, and V. Vardanyan, Dark energy, $\alpha$-attractors, and large-scale structure surveys, J. Cosmol. Astropart. Phys. 06 (2018) 041.

[18] M. Atkins and X. Calmet, Bounds on the Nonminimal Coupling of the Higgs Boson to Gravity, Phys. Rev. Lett. 110, 051301 (2013).

[19] J. R. Espinosa, Cosmological implications of Higgs nearcriticality, Phil. Trans. R. Soc. A 376, 20170118 (2018).

[20] T. Markkanen, A. Rajantie, and S. Stopyra, Cosmological aspects of Higgs vacuum metastability, arXiv:1809.06923.

[21] P. Agrawal, G. Obied, P. J. Steinhardt, and C. Vafa, On the cosmological implications of the string Swampland, Phys. Lett. B 784, 271 (2018).

[22] A. Achúcarro and G. A. Palma, Bounds on slow roll and the de Sitter Swampland, arXiv:1807.04390.

[23] S. K. Garg and C. Krishnan, The string swampland constraints require multi-field inflation, arXiv:1807.05193.
[24] S. Brahma and M. Wali Hossain, Avoiding the string swampland in single-field inflation: Excited initial states, arXiv: 1809.01277.

[25] S. Das, A note on single-field inflation and the swampland Criteria, arXiv:1809.03962.

[26] C.-M. Lin, K.-W. Ng, and K. Cheung, Chaotic inflation on the brane and the swampland criteria, arXiv:1810.01644.

[27] M. Motaharfar, V. Kamali, and R. O. Ramos, Warm way out of the Swampland, arXiv:1810.02816.

[28] A. Ashoorioon, Rescuing single field inflation from the Swampland, arXiv:1810.04001.

[29] S. Das, Warm inflation in the light of swampland criteria, arXiv: 1810.05038 .

[30] J.-L. Lehners, Small-field and scale-free: Inflation and ekpyrosis at their extremes, J. Cosmol. Astropart. Phys. 11 (2018) 001.

[31] M. Dias, J. Frazer, A. Retolaza, and A. Westphal, Primordial gravitational waves and the Swampland, arXiv: 1807.06579.

[32] F. Denef, A. Hebecker, and T. Wrase, de Sitter swampland conjecture and the Higgs potential, Phys. Rev. D 98, 086004 (2018).

[33] E. Colgáin, M. H. P. M. Van Putten, and H. Yavartanoo, H0 tension and the de Sitter Swampland, arXiv:1807.07451.

[34] H. Matsui and F. Takahashi, Eternal inflation and swampland conjectures, arXiv:1807.11938.

[35] I. Ben-Dayan, Draining the Swampland, arXiv:1808.01615.

[36] C.-I. Chiang and H. Murayama, Building supergravity quintessence model, arXiv:1808.02279.

[37] L. Heisenberg, M. Bartelmann, R. Brandenberger, and A. Refregier, Dark energy in the Swampland, Phys. Rev. D 98, 123502 (2018).

[38] W. H. Kinney, S. Vagnozzi, and L. Visinelli, The Zoo plot meets the Swampland: Mutual (in)consistency of singlefield inflation, string conjectures, and cosmological data, arXiv:1808.06424.

[39] C. Han, S. Pi, and M. Sasaki, Quintessence saves Higgs instability, arXiv:1809.05507.

[40] S. D. Odintsov and V. K. Oikonomou, Finite-time singularities in Swampland-related dark energy models, arXiv: 1810.03575 .

[41] M. Reece, Photon masses in the landscape and the Swampland, arXiv:1808.09966.

[42] L. Heisenberg, M. Bartelmann, R. Brandenberger, and A. Refregier, Dark energy in the swampland II, arXiv:1809 .00154 .

[43] H. Murayama, M. Yamazaki, and T. T. Yanagida, Do we live in the Swampland?, J. High Energy Phys. 12 (2018) 032.

[44] M. C. D. Marsh, The Swampland, quintessence and the vacuum energy, arXiv:1809.00726.

[45] K. Choi, D. Chway, and C.S. Shin, The dS swampland conjecture with the electroweak symmetry and QCD chiral symmetry breaking, J. High Energy Phys. 11 (2018) 142.

[46] H. Matsui, F. Takahashi, and M. Yamada, Isocurvature perturbations of dark energy and dark matter from the swampland conjecture, arXiv:1809.07286.

[47] K. Hamaguchi, M. Ibe, and T. Moroi, The swampland conjecture and the Higgs expectation value, J. High Energy Phys. 12 (2018) 023. 
[48] M. Kawasaki and V. Takhistov, Primordial black holes and the string Swampland, Phys. Rev. D 98, 123514 (2018).

[49] U. H. Danielsson and T. Van Riet, What if string theory has no de Sitter vacua?, Int. J. Mod. Phys. D 27, 1830007 (2018).

[50] D. Andriot, On the de Sitter swampland criterion, Phys. Lett. B 785, 570 (2018).

[51] A. Kehagias and A. Riotto, A note on inflation and the Swampland, arXiv:1807.05445.

[52] C. Roupec and T. Wrase, de Sitter extrema and the Swampland, Fortschr. Phys. 1800082 (2018).

[53] D. Andriot, New constraints on classical de Sitter: Flirting with the Swampland, arXiv:1807.09698.

[54] J. P. Conlon, The de Sitter swampland conjecture and supersymmetric AdS vacua, Int. J. Mod. Phys. A 33, 1850178 (2018).

[55] K. Dasgupta, M. Emelin, E. McDonough, and R. Tatar, Quantum corrections and the de Sitter swampland conjecture, arXiv:1808.07498.

[56] M. Cicoli, S. De Alwis, A. Maharana, F. Muia, and F. Quevedo, De Sitter vs quintessence in string theory, Fortschr. Phys. 1800079 (2018).
[57] Y. Akrami, R. Kallosh, A. Linde, and V. Vardanyan, The landscape, the swampland and the era of precision cosmology, Fortschr. Phys. 1800075 (2018).

[58] I. Bena, E. Dudas, M. Graña, and S. Lüst, Uplifting runaways, Fortschr. Phys. 1800100 (2018).

[59] S. Dimopoulos and H. Georgi, Softly broken supersymmetry and SU(5), Nucl. Phys. B193, 150 (1981).

[60] L. Randall and R. Sundrum, A large mass hierarchy from a small extra dimension, Phys. Rev. Lett. 83, 3370 (1999).

[61] N. Arkani-Hamed, S. Dimopoulos, and G. R. Dvali, The Hierarchy problem and new dimensions at a millimeter, Phys. Lett. B 429, 263 (1998).

[62] S. Weinberg, Implications of dynamical symmetry breaking, Phys. Rev. D 13, 974 (1976); 19, 1277(A) (1979).

[63] L. Susskind, Dynamics of spontaneous symmetry breaking in the Weinberg-Salam theory, Phys. Rev. D 20, 2619 (1979).

[64] P. W. Graham, D. E. Kaplan, and S. Rajendran, Cosmological Relaxation of the Electroweak Scale, Phys. Rev. Lett. 115, 221801 (2015).

[65] N. Arkani-Hamed, T. Cohen, R. T. D’Agnolo, A. Hook, H. D. Kim, and D. Pinner, Solving the Hierarchy Problem at Reheating with a Large Number of Degrees of Freedom, Phys. Rev. Lett. 117, 251801 (2016). 